\section{Forscher für die Zukunft starten wieder durch}

Nach einer Corona-bedingten Auszeit 2020 sind die Forscher für die Zukunft dieses Jahr mit einem fulminanten Auftakt an der Berliner Charité wieder voll durchgestartet. 2 Tage lang ging es um die radiologische Forschung, Interventionsradiologie und mögliche Karrierewege der teilnehmenden Nachwuchsforschenden aus ganz Deutschland.

Für den ersten Tag hat das Team der Charité um Professor Bernd Hamm die Forschung in den programmatischen Fokus gesetzt. Es wurde von praktisch Erfahrenen beschrieben, wie man gute Fördermittelanträge schreibt, was es für Fördermittelgeber gibt und wie die Entscheidungsprozesse ablaufen. Ein weiterer Schwerpunkt war die Vorstellung des deutschlandweiten RACOONNetzwerkes. Am Nachmittag wurden einzelne Forschungsfelder anhand von Handson-Übungen in Kleingruppen beleuchtet:

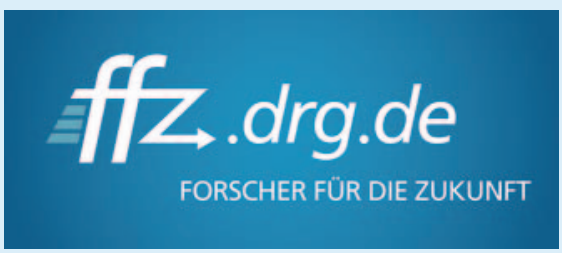

Geboten wurden ein Einblick in die Elastografie, Rheuma-Cases, eine Live-Demo aus dem experimentellen Tierlabor und eine praktische Übung am Angio-Trainer.

Am zweiten Tag wurden die Teilnehmenden in die Möglichkeiten der interventionsradiologischen Forschung eingeführt. Hier standen klinische und translationale Studien im Vordergrund. Aber auch der weitere Weg hin zu einem konkreten Medizinprodukt und der damit möglichen Ausgründung wurde im Sinne einer auf Forschung aufbau- enden Karriere besprochen und Vorbilder gezeigt.

Die nächsten beiden Stationen der aktuellen Staffel sind dann im Februar in Würzburg und im März in Göttingen.

Neben der Vertiefung fachlicher Aspekte ist die Vernetzung der herausragenden wissenschaftlichen Nachwuchskräfte ein wesentliches Ziel des FFZ-Programms. Bei den Workshops geknüpfte Kontakte können bei regelmäßigen Treffen der FFZ-Teilnehmerinnen und -Teilnehmer oder dem ECR weiter gepflegt werden. Darüber hinaus bietet die AG Methodik und Forschung (AGMF) ein Forum für alle an radiologischer Forschung interessierten Mitglieder der DRG.

Weitere Informationen erhalten Sie unter www.ffz.drg.de 